\title{
The Cosmological Constant Problem and Quantum Spacetime Reference Frame
}

\author{
M.J.Luo ${ }^{1, *}$ \\ ${ }^{1}$ Department of Physics, Jiangsu University, Zhenjiang 212013, People's Republic of China
}

\begin{abstract}
We generalize the idea of quantum clock time to quantum spacetime reference frame via physical realization of a reference system by quantum rulers and clocks. Omitting the internal degrees of freedom (such as spins) of the physical rulers and clocks, only considering their metric properties, the spacetime reference frame is described by a bosonic non-linear sigma model. We study the quantum behavior of the system under approximations, and obtain (1) a cosmological constant valued $(2 / \pi) \rho_{c 0}$ ( $\rho_{c 0}$ the critical density at near current epoch) which is very close to the observations; $(2)$ an effective Einstein-Hilbert term in the effective action; (3) the ratio of variance to mean-squared of spacetime interval tends to a universal constant $2 / \pi$ in the infrared region. This effect is testable by observing a linear dependence between the inherent quantum variance and mean-squared of the redshifts from cosmic distant spectral lines. The proportionality is expected to be the observed percentage of the dark energy. We also generalize the equivalence principle to be valid for all quantum phenomenon.
\end{abstract}

\section{INTRODUCTION}

Reference frame is one of the most fundamental notions in physics. When a measurement in physics is performed or described, a reference frame has always been explicitly or implicitly used. As ordinarily formulated, reference frame idealizationally uses the rulers and clocks to label the spacetime for simplicity, which have well-defined values of coordinates and are considered most perfect, absolute, classical, and external. This fundamentally classical notion of reference frame has been using in almost all area of physics including today's textbook quantum physics, although quantum mechanics has been discovered for a century. The quantum mechanics tells us that all measuring devices are subject to some level of quantum fluctuations, certainly applying to the rulers and clocks, namely the spacetime. Such idealizational treatment works well in quantum mechanics and quantum field theory when the equations of them cast in terms of the variables that are really measured by physical rulers and clocks in ordinary laboratory. This is, to a large extent, due to the fact that gravitational effects are not seriously taken into account in most laboratory experiments. Since according to the standard theory of gravity, the general relativity, the spacetime is dynamical and relational. It is as expected, when the quantum mechanics is applied to the cosmology which is gravity dominated, severe difficulty arises: the cosmological constant problem, see for instance [1-3] and references therein.

Along this line of thinking, treating the concept of a reference frame in quantum theory may be the key to the cosmological constant problem. The earlier publications $[4,5]$ have proposed a possible solution by replacing the idealized parameter time in textbook quantum theory by a quantum dynamical variable playing the role of a physical clock time. The papers obtain a cosmological constant having not only a correct order but also a percentage $\Omega_{\Lambda}=$ $2 / \pi \approx 0.64$ which is very close to current observational value [6]. However, such solution can only be regarded as incomplete, since although time has been treated quantum mechanically, in those papers, the spatial coordinates are still treated as classical external parameters free from quantum fluctuations. The space and time measurements are closely related to each other and must be treated on an equal footing. In this work, we shall generalize the discussion of quantum clock time in refs. $[4,5]$ to a more general framework: quantum spacetime reference frame. It is a years old idea (see for instance [7-10] and references therein), but to the best of our knowledge, there is no literature or discussion yet connecting such idea to the cosmological constant problem, the goal of the paper is to show their profound relation. We take the natural unit $\hbar=c=1$ for convention in the paper.

\section{QUANTUM PHYSICAL SYSTEM RELATIVE TO A QUANTUM REFERENCE FRAME}

When a reference frame (rulers, clocks or other measuring devices), to which a to-be-studied quantum physical system is relative, are inherent quantum, they can not be just ignored from a complete quantum description. In a complete quantum treatment, a set of states of the to-be-studied quantum physical system (denoted by P) together with the quantum reference frame system (denoted by $\mathrm{R}$ ) are described by the Hilbert space $\mathcal{H}$ being a direct product

*Electronic address: mjluo@ujs.edu.cn 
of both Hilbert spaces,

$$
\mathcal{H}=\mathcal{H}_{P} \otimes \mathcal{H}_{R}
$$

However, it does not mean that the state vector of the whole system is simply a direct product of vector in each Hilbert space. In most cases, it is an entangled state, the reason is as follow. In the first step of performing a physical measurement, instrument calibration must be carried out firstly. A good experimental calibration establishes a oneto-one correlation between a state of the to-be-studied quantum physical system $|P\rangle_{i}$ and a state of the measuring device $|R\rangle_{j}$. If the calibration is well done, such step introduces a complete bases $|P\rangle_{i} \otimes|R\rangle_{j}$ to expand a state $|\Psi\rangle$ in the whole Hilbert space $\mathcal{H}$,

$$
|\Psi\rangle=\sum_{i, j} \alpha_{i j}|P\rangle_{i} \otimes|R\rangle_{j}
$$

In the second step, physicist let these two systems evolve independently and observe the output of the measuring device. The physics of the individual measuring device is assumed well understood, having complete knowledge of the Hilbert space $\mathcal{H}_{R}$, and a state in it can be expanded by a complete bases $|R\rangle=\sum_{j} \beta_{j}|R\rangle_{j}$. Then physicist uses the information of calibration Eq.(2) to judge what is the state of $\mathrm{P}$ when the output of the measuring device being in one of the state $|R\rangle_{j}$. The expansion coefficient $\alpha_{i j}$ measures the amplitude of the measuring device being in the state $|R\rangle_{j}$, and meanwhile, the to-be-studied system $\mathrm{P}$ being in the state $|P\rangle_{i}$. The $\left|\alpha_{i j}\right|^{2}$ measures the joint probability that $\mathrm{P}$ and $\mathrm{R}$ are in the states $|P\rangle_{i}$ and $|R\rangle_{j}$ respectively.

\section{A. Relational Interpretation}

The entangled state has a general property that the amplitude $\alpha_{i j}$ can not be factorized into a product of each amplitude of $|P\rangle,|R\rangle$, namely, for all amplitudes $\gamma_{i}, \beta_{j}$, defined by $|P\rangle=\sum_{i} \gamma_{i}|P\rangle_{i}$ and $|R\rangle=\sum_{j} \beta_{j}|R\rangle_{j}$, we always have $\alpha_{i j} \neq \gamma_{i} \beta_{j}$. This property has important physical implications.

A physical meaningful probability in a measurement is the probability of state $|P\rangle_{i}$ given the condition that the measuring device is observed in the state $|R\rangle_{j}$. The conditional probability $\operatorname{Pro}\left(P_{i} \mid R_{j}\right)$ is defined by

$$
\operatorname{Pro}\left(P_{i} \mid R_{j}\right) \equiv \frac{\operatorname{Pro}\left(P_{i} \cap R_{j}\right)}{\operatorname{Pro}\left(R_{j}\right)}=\frac{\left|\alpha_{i j}\right|^{2}}{\left|\beta_{j}\right|^{2}},
$$

in which the $\operatorname{Pro}\left(P_{i} \cap R_{j}\right)$ stands for the joint probability $\left|\alpha_{i j}\right|^{2}$. The $\operatorname{Pro}\left(R_{j}\right)$, which is given by $\left|\beta_{j}\right|^{2}$, represents the probability of state $|R\rangle_{j}$ when the measuring device independently evolves in the second step. Since $\alpha_{i j} \neq \gamma_{i} \beta_{j}$, the conditional probability that $\mathrm{P}$ is in $|P\rangle_{i}$ given $\mathrm{R}$ being in $|R\rangle_{j}$ does not equal to the probability that $\mathrm{P}$ is individually in the state $|P\rangle_{i}$, i.e. $\operatorname{Pro}\left(P_{i} \mid R_{j}\right) \neq \operatorname{Pro}\left(P_{i}\right)$. In this sense, the probability of $|P\rangle_{i}$ is affected by the probability distribution of $|R\rangle_{j}$. The state $|P\rangle_{i}$ of the to-be-studied system has no individual absolute meaning, it makes sense only relative to the state $|R\rangle_{j}$ of the measuring device as a reference [11, 12], namely, the state is relational [13, 14].

The entangled state is purely a quantum state, having no classical correspondence. Only when the measuring devices are treated semi-classically, i.e. a delta distribution for $|R\rangle_{j}$, the sign of inequality becomes an equal sign, in this limit it recovers the absolute probability interpretation of the textbook quantum mechanics. For instance, in general the distribution for $|R\rangle_{j}$ has a finite width due to quantum fluctuation, one can prove that it is equivalent to a textbook wavefunction of $\mathrm{P}$ with a smeared variable $\mathrm{R}[15]$.

In this paper, we argue that when a quantum theory is completely formulated in terms of the states entangling a tobe-studied quantum physical system with a quantum reference frame or quantum measuring devices, and interpreted it in a relational manner, the quantum theory is able to accommodate the spirit of relativity, leading to a consistent theory of quantum spacetime reference frame.

\section{B. Identical Particles Model of Spacetime Reference Frame}

To take a further look into the Hilbert space of the reference frame $\mathcal{H}_{R}$, let us considering an operative setup to realize the physical rulers and clocks at the quantum level. Suppose we have $D$ free identical scalar particles, living in a $d$-dimensional background $x_{a},(a=0,1,2, \ldots, d-1)$. Omitting the internal degrees of freedom of them such as spin, let $X_{\mu}(x),(\mu=0,1,2, \ldots, D-1)$ represent scalar fields of these identical particles, with dimension [mass $]^{-1}$. 
As ordinarily described, the frame $x$ can be interpreted as the coordinates according to the walls and clock of a laboratory. Assuming that these identical scalar fields evolve independently in the laboratory, the resulting action is the summation of each particle, namely, the action is separable,

$$
S_{R}[X]=\sum_{\mu}^{D} S_{i d}\left[X_{\mu}\right]
$$

The action is separable means that the Hilbert space of the identical particles system is a direct product of each particles $\mathcal{H}_{R}=\otimes_{\mu}^{D} \mathcal{H}_{X_{\mu}}$, however, as is shown below, for the nontrivial interchange properties, the state of the identical particles or equivalently the spacetime reference frame is also an entangled state of each particle, but rather a simply direct product. For each identical particles, $S_{i d}\left[X_{\mu}\right]$ is a standard free scalar field action formulated in a homogenous and flat frame with respect to the laboratory walls and clock

$$
S_{i d}\left[X_{\mu}\right]=\frac{\lambda}{2} \int d^{4} x \partial_{a} X_{\mu} \partial_{a} X_{\mu}, \quad(\mu=0,1,2,3)
$$

in which $\partial_{a}=\frac{\partial}{\partial x_{a}}$, and $\lambda$ is a constant having dimension [mass] $]^{d}$ and related to a "rest mass" scale (with respect to the frame $x$ ) of the identical scalar particles, since we have used the laboratory wall interpretation to $x_{a}$ so here $d=4$.

In a range of ordinary laboratory scale, the walls and clock of the laboratory can be used to orient, align and order the beams of these scalar particles at high precision, in this sense, these identical quantum scalar free fields can be interpreted as physical rulers and clock at the quantum level. For example, the identical scalar fields oriented as $X_{1,2,3}=X, Y, Z$ can be aligned with a reference to the $x, y, z$-directions of the walls of the laboratory respectively. One could visualize them as local quantum vibrations or oscillations placed on the lattice of $x, y, z$, these identical particles can be seen as rulers since distances are able be measured by counting their phase changes of the local vibrations if events trigger the counting. In this sense they play the roles of state-triggers labeling where the event happens. For the same consideration, a scalar field denoted as $X_{0}=T$ on the lattice can be used to play the role of a small pendulum clock labeling the causal order of the events, i.e. when the event happens. As a result, an event denoted by state $|P\rangle_{i}$ is entangled with a particular configuration of the identical particles system it triggers

$$
|R\rangle_{j}=\left|X_{0}, X_{1}, X_{2}, X_{3}\right\rangle
$$

according to the entanglement Eq.(2). So we could say that a state of the identical particles as a standard reference system labels where and when a quantum event happens, playing the role of a spacetime reference frame.

For the bosonic statistics of these four identical particles, the interchange between them does not change the state $|R\rangle_{j}$. In one case, because the role of the vibrations on the lattice as ruler or clock is just a convention, so if we interchange $X_{0}$ and $X_{i},(i=1,2,3)$ the system obviously does not change. In another case, if we interchange two rulers' fields, for instance, $X$ and $Y$, the frame changes from left-hand to right-hand, but we have the state unchanged, i.e. $\left|X_{0}, X_{1}, X_{2}, X_{3}\right\rangle=\left|X_{0}, X_{2}, X_{1}, X_{3}\right\rangle$, which implies a reflection symmetry (the parity symmetry) of spacetime coordinates. In general, the configuration space of the identical particles is $\mathbb{R}^{4} / S_{4}$, where $S_{4}$ is the permutation symmetry of the identical particles representing the discrete symmetries of spacetime and/or their certain products.

A practical example for the identical scalar particles model of a spacetime reference frame is the multi-wire chamber. The beams of the scalar fields used in the model can be considered as free electron fields in the array of multi-wire, which signal the coordinates of an event by an impulse at the output. As it is pointed out, the original electron signal triggered by the event is inescapably quantum, more precisely, the electron obeys the quantum uncertainty principle.

When the scale of an experimental measurement is much larger than the scale of an ordinary laboratory, the laboratory wall interpretation becomes no longer valid, since the distance can not be measured directly as the situation in the laboratory. What we could use to measure spacetime coordinates is inevitably only by using the identical particles being our rulers and clock. For example, in the situation of a cosmic observation, the scalar particles used in the model can be instead considered as free photons. The information of distance of an event can only be extracted from observations of, e.g. luminosity and frequency/redshift of distant spectral lines.

Here the notion of metric is in general non-trivial in the situation that the spacetime coordinates is operationally measured by the physical fields as rulers and clock. Since a realistic geometry measured via the free fields in general does not necessarily match the geometry which expected beyond the laboratory. For example, a ruler or clock elsewhere measured by a field $X_{i}$ or $X_{0}$ requires certain technique to compare or synchronize to the laboratory ruler $x_{a}$ or clock $x_{0}$, regardless the laboratory rulers and clock are realistic or extrapolative or complete imaginary in human mind. Briefly speaking, the identical particles propagates in a general curved spacetime measured by themselves. By using mathematical method, the metric of the actual spacetime can be given by a vierbein, which makes a comparison between the physical coordinates $X_{\mu}$ and the extrapolated absolute coordinates $x_{a}, e_{\mu}^{a} \equiv \partial X_{\mu} / \partial x_{a}$. The metric 
having dimension $[\text { mass }]^{0}$ is defined by

$$
g_{\mu \nu}(x)=e_{\mu}^{a} e_{\nu}^{a}=\frac{\partial X_{\mu}(x)}{\partial x_{a}} \frac{\partial X_{\nu}(x)}{\partial x_{a}},
$$

in which the extrapolated laboratory wall frame $x$ is assumed homogeneous and flat as well. The assumption is considered having no impact on the physical result since the physics does not depend on the choice of frame, which can seen more clearly later. Precisely speaking, we can not align and order the beams of these identical free scalar particles according to the walls and clock of the laboratory in prior, but in general according to the metric $g_{\mu \nu}=\left(g^{\mu \nu}\right)^{-1}$ practically measured. In this situation, the action of identical particles are generalized to

$$
S_{R}[X]=\frac{\lambda}{2} \int d^{d} x \sum_{\mu}^{D} \partial_{a} X^{\mu} \partial_{a} X_{\mu}=\frac{\lambda}{2} \int d^{d} x \sum_{\mu, \nu}^{D} g_{\mu \nu} \partial_{a} X^{\mu} \partial_{a} X^{\nu}
$$

where the indices are raised or lowered by the metric, e.g. $X^{\mu}=g^{\mu \nu} X_{\nu}$. In the action the configuration space of the identical particles locally is generalized from $\mathbb{R}^{4}$ to a more general Riemannian space $\left(\mathbb{M}^{4}, g\right)$ with non-trivial metric, and we do not pre-assume the value of dimension $d$ from the beginning whose value will be discussed in detail later.

For the reason that a $D=4$ curved manifold can be isometrically embedded in a linear space of $D+1$ dimensions, one can also start from a linear theory without prior geometry notion such as metric

$$
S[\phi]=\int d^{d} x\left[\frac{\lambda}{2} \sum_{\mu=1}^{D+1} \partial_{a} \phi^{\mu} \partial_{a} \phi^{\mu}+\frac{1}{2} i \xi\left(\sum_{\mu=1}^{D+1} \lambda \phi^{\mu} \phi^{\mu}-M^{d-2}\right)\right],
$$

in which $\xi$ is a Lagrangian multiplier that imposes the constraint $\sum_{\mu=1}^{D+1} \lambda \phi^{\mu} \phi^{\mu}=M^{d-2}$, where $M$ is considered a foundamental energy scale. When we functionally integrate out $\xi$ with the identification $\phi=\left(\delta X^{0}, \delta X^{1}, \delta X^{2}, \delta X^{3}, \sigma\right)$, Eq.(9) can be formulated in the form of Eq.(8)

$$
S[\delta X]=\frac{\lambda}{2} \int d^{d} x \sum_{\mu, \nu}^{D}\left(\eta_{\mu \nu}+\frac{\delta X_{\mu} \delta X_{\nu}}{\sigma^{2}}\right) \partial_{a}\left(\delta X^{\mu}\right) \partial_{a}\left(\delta X^{\nu}\right),
$$

with an induced metric

$$
\tilde{g}_{\mu \nu}[X]=\eta_{\mu \nu}+\frac{\delta X_{\mu} \delta X_{\nu}}{\sigma^{2}}
$$

The induced metric Eq.(11) is equivalent to the expectation value of the metric Eq.(7), since according to the equivalence principle we can always choose a local inertial reference frame such that the spacetime $X_{\mu}$ seems to be locally the flat parameter background $x$ (the laboratory wall frame), if we expand $X_{\mu}$ in Eq.(7) around the background $x$ with fluctuations $X_{\mu}(x)=x_{\mu}+\delta X_{\mu}(x)$ for any chosen point $x$, taking $\left\langle\delta X_{\mu}\right\rangle=0$ while $\left\langle\delta X_{\mu} \delta X_{\nu}\right\rangle \neq 0$, and considering field value $\sigma$ measures the proper length $\left(\sum_{a} \delta x_{a}^{2}\right)^{1 / 2}$ of the parameter background. Furthermore, as the proper length of the parameter background hardly vary with spacetime, the $\sigma$ field is therefore too massive to have any fluctuations and excited states, the $\sigma$ field is merely an unobserved auxiliary field, what we used to measure the realistic spacetime are thoes identical particles $X_{\mu}$.

For historic reasons, the action Eq.(8) is known as the non-linear sigma model (NLSM) [16-18] having many applications in particle physics and condensed matter physics. The action Eq.(8) maps a d-dimensional flat absolute parameter background $x_{a}$ into a $D$-dimensional target Riemannian manifold $X_{\mu}$ which is the configuration space of the identical particles. The target manifold is invariant under local $O(D)$ rotation symmetry, $X^{\mu} \rightarrow X^{\mu \prime}=\Lambda_{\nu}^{\mu} X^{\nu}$. Such compact symmetry can be easily transformed into the noncompact $O(1, D-1)$ symmetry by an unimportant phase reversal (a $\pi$-phase shift) redefinition to the clock field $X^{0}$, i.e. $X^{0} \rightarrow i X^{0}$ when we initialize the field. The $O(D)$ or $O(1, D-1)$ symmetry can be interpreted as the Lorentz symmetry of the spacetime reference frame.

An important point must be emphasized here is that in the rest of the paper the absolute parameter background $x_{a}$ previously interpreted as an extrapolative absolute laboratory wall frame will have nothing to do with the realistic spacetime. A parameter background is necessary for the theoretical description of a quantum fields theory, but they are not necessarily interpreted as the physical spacetime, the realistic physical spacetime is what we measure from the identical scalar fields $X^{\mu}$ being the standard reference system. This point is very important in resolving the cosmological constant problem which will be shown in subsection-D of the section III.

Notice that the classical Lagrangian of NLSM is formally proportional to $g_{\mu \nu} g^{\mu \nu}=D$, it is the dimension of the target manifold which is an invariant under the parameter background $x$-coordinates transformations, so the NLSM is 
parameter background independent which is the reason we could choose a flat background without loss of any physical generality. Furthermore, the action is also invariant under an arbitrary differentiable $X^{\mu}$-coordinates transformation of the target manifold. In this sense, a system relative to such spacetime reference frame is connected to a theory of gravity on the target manifold, their relationship will be shown in the next section.

A choice of a reference $\mathcal{H}_{R}$ is mathematically equivalent to choose a complete set of bases to formulate the whole Hilbert space $\mathcal{H}=\mathcal{H}_{P} \otimes \mathcal{H}_{R}$. More precisely, a choice of a spacetime reference frame explicitly solves the diffeomorphism constraints [8] and the Wheeler-DeWitt equation. The constraints say that physics dose not depend on the choice of reference frame, so if you choose one frame, a physical state must be a summation over all possible choices. In this sense, it again indicates that a physical state in $\mathcal{H}$ is not a simple direct product state, it is an entangled state as Eq.(2) summing over all possible direct product states.

\section{Semi-Classical Approximation}

In this subsection we will take a first look at the whole system consisting of a to-be-studied quantum physical system $\mathrm{P}$ and a quantum spacetime reference frame $\mathrm{R}$ which $\mathrm{P}$ relative to. After the calibration, the entanglement between $\mathrm{P}$ and $\mathrm{R}$ is developed. Then $\mathrm{P}$ and $\mathrm{R}$ evolve independently, and we assume that they do not interact, so the total action can be separably written as

$$
S[\varphi, X]=S_{P}[\varphi]+S_{R}[X]
$$

Without loss of generality, we consider the to-be-studied system is a scalar fields theory formulated in the same laboratory wall frame as that formulating the spacetime reference system Eq.(8),

$$
S_{P}[\varphi]=\int d^{d} x\left(\frac{1}{2} \partial_{a} \varphi \partial_{a} \varphi-V(\varphi)\right)
$$

So that the reference frame $\mathrm{R}$ and the to-be-studied system $\mathrm{P}$ share the same laboratory coordinates $x$, the total action is then given by

$$
S[\varphi, X]=\int d^{d} x\left[\frac{1}{2} \partial_{a} \varphi \partial_{a} \varphi-V(\varphi)+\frac{\lambda}{2} g_{\mu \nu} \partial_{a} X^{\mu} \partial_{a} X^{\nu}\right],
$$

in which we have neglected the constraint of the NLSM. In a semi-classical approximation in which the fields $X_{\mu}$ can be seen as parameters, the action can be rewritten as

$$
S_{e f f}[\varphi]=\int d^{4} X \sqrt{\operatorname{det} g}\left[\frac{1}{4}\left\langle g_{\mu \nu} \partial_{a} X^{\mu} \partial_{a} X^{\nu}\right\rangle\left(\frac{1}{2} g^{\mu \nu} \frac{\delta \varphi}{\delta X^{\mu}} \frac{\delta \varphi}{\delta X^{\nu}}+2 \lambda\right)-V(\varphi)\right]
$$

where we have used $g_{\mu \nu} g^{\mu \nu}=D=4$ and $\sqrt{\operatorname{det} g}=\left\|\frac{\partial x_{a}}{\partial X^{\mu}}\right\|$ is the Jacobian determinant changing the integration variable from $x$ to $X$. The Jacobian matrix is a square matrix, the property requires the dimension of the space $x$ equaling to the number of the identical particles $X$, so $d=D=4$. It must be emphasized that this statement is true only in the semi-classical approximation, it is not necessarily true beyond the approximation as will be shown in the next section when we study its quantum behavior. Since $\left\langle g^{\mu \nu}\right\rangle=\left\langle\partial_{a} X^{\mu} \partial_{a} X^{\nu}\right\rangle$, so $\frac{1}{4}\left\langle g_{\mu \nu} \partial_{a} X^{\mu} \partial_{a} X^{\nu}\right\rangle=1$. We obtain

$$
S_{e f f}[\varphi]=\int d^{4} X \sqrt{\operatorname{det} g}\left[\frac{1}{2} g^{\mu \nu} \frac{\delta \varphi}{\delta X^{\mu}} \frac{\delta \varphi}{\delta X^{\nu}}-V(\varphi)+2 \lambda\right] .
$$

The effective action obtained from the semi-classical approximation has a straightforward interpretation: the tobe-studied quantum fields system of $\varphi$ is relative to the spacetime reference frame $X^{\mu}$ semi-classically treated. The equation Eq.(16) is similar with Eq.(13) up to an unimportant constant, just formally the derivative $\frac{\partial}{\partial x}$ is replaced by a functional derivative $\frac{\delta}{\delta X}$, and the function $\varphi(x)$ with respect to $x$ is replaced by a functional $\varphi[X]$ with respect to $X$.

Furthermore, although parameter background space $x$ is flat, when the target manifold is curved, the effective action includes such a theory that field or even quantized field $\varphi$ is in a curved spacetime. So we prove the equivalence between the semi-classical theory of the spacetime reference frame and the theories of (quantum) fields in curved spacetime. Thus it is reasonable to expect that the theory could recover the existing results of quantum fields theories in curved spacetime, for instance the Hawking's radiation of a black hole.

However, only a semi-classical treatment of the spacetime reference frame is not enough, where the spacetime is still fix, the quantum dynamics of the spacetime reference frame must be considered. We will show in the next section that an effective Einstein's theory of gravity and a correct cosmological constant naturally arise from the quantum behavior of the spacetime reference frame. 


\section{QUANTUM BEHAVIOR}

In this section, we will study the quantum dynamics of the spacetime reference frame defined in previous section. Let us recall the NLSM in Eq.(8), which maps a $d$ dimensional flat homogeneous parameter background $x_{a},(a=$ $0,1,2, \ldots, d-1)$ into a $D$-dimensional target manifold $X^{\mu},(\mu=0,1,2, \ldots, D-1)$. Here we interpret the target manifold coordinates $X^{\mu}$ as scalar fields describing the coordinates of the realistic spacetime, so $D \equiv 4$ is considered fixed. However, it does not necessary to fix the dimension $d$ of parameter background from the beginning, since first it is unobservable in the theory and second it is known that $d$ runs as renormalization flows, the discrepancy is known as the anomalous dimension. Therefore here the parameter background space has nothing to do with our realistic spacetime any more in the theory, and $d$ is considered varying with the scale of renormalization. The action of the NLSM is

$$
S_{N L S M}=\frac{\lambda}{2} \int d^{d} x g_{\mu \nu}[X] \partial_{a} X^{\mu} \partial_{a} X^{\nu}
$$

where $g_{\mu \nu}[X]$ is a positive dimensionless metric of the target manifold, and since coordinates $x_{a}$ and $X^{\mu}$ have dimensions $[\mathrm{mass}]^{-1}$, the constant $\lambda$ has dimension $[\mathrm{mass}]^{d}$.

\section{A. Renormalization}

Most of the quantum behaviors of a system encode in its renormalization. For the sake of making the problem concerning the cosmological constant problem as simple as possible, we only discuss an effective field renormalization function $Z(k)=1+\delta_{Z}(k)$, which rescales each field isotropically,

$$
X^{\mu} \rightarrow X_{r e}^{\mu}=Z^{1 / 2}(k) X^{\mu} .
$$

The parameter $k$, with dimension $[\text { mass }]^{1}$, introduced by hand measures a cutoff of the Fourier component of the dynamical spacetime fields $X^{\mu}$. So here $k$ replaces the absolute parameter background $x$, playing the role of a renormalization evolution parameter. Then the action in a "Wilsonian" sense is effective defined at the cutoff $k$,

$$
S_{k}=\frac{\lambda}{2} \int d^{d} x Z g_{\mu \nu} \partial_{a} X^{\mu} \partial_{a} X^{\nu}
$$

The renormalization function $Z$ can also be interpreted as a renormalization to the constant $\lambda$ or $g_{\mu \nu}$ while $X^{\mu}$ fixed, which is a completely different physical interpretation but in our discussions the mathematics is the same.

Recall that, in the semi-classical approximation, the dimensions of the parameter background is identical with that of the target manifold, i.e. $d=D \equiv 4$. Although it seems that the NLSM in $d=4$ is perturbative non-renormalizable by power counting, but at the non-perturbative level, it is shown [19] that a $d=4$ NLSM has a non-trivial (nonGaussian) UV fixed point (i.e. $k \rightarrow \infty$ ). In this sense, this theory of quantum spacetime is "asymptotically safe" [20], which will be clearly seen later by noting that $d$ runs to 2 at $k \rightarrow \infty$. Therefore, the fields $X^{\mu}$ and $\lambda$ in Eq.(17) can be seen as bare values defined at the UV fixed point, at which the renormalization condition is written as

$$
\lim _{k \rightarrow \infty} Z(k)=1, \quad \lim _{k \rightarrow \infty} \delta_{Z}(k)=0 .
$$

In order to find the physics at IR, the question we want to ask is: from this initial renormalization condition at UV, what value does it take when the renormalization group flows to $\operatorname{IR}(k \rightarrow 0)$ ?

The answer to the question for the $d=4$ NLSM has been studied in literature by perturbation theory when the value of $\lambda$ is large [19] (the coupling of the NLSM being the inverse of $\lambda$ ). Fortunately in our theory the bare value $\lambda$ defined at UV is indeed large. It is of order $\lambda \sim \mathcal{O}\left(R_{*} k_{U V}^{d-2}\right)$ [19], where $R_{*}$ is a positive Ricci scalar curvature of the target manifold at a fixed point and $k_{U V}$ is a constant relating to a UV scale much larger than the scale $k$ we are interested in, i.e. $k_{U V} \gg k$.

In this situation, a large $\lambda$ corresponds to a small coupling, the perturbation calculation of $\delta_{Z}$ is reliable, which at one loop is given by

$$
\delta_{Z}(k)=\frac{1}{2} \frac{R}{\lambda D} \int_{0 \leq|p|<k} \frac{d^{d} p}{(2 \pi)^{d}} \frac{i}{p^{2}}=\frac{1}{(4 \pi)^{d / 2} \Gamma\left(\frac{d}{2}\right)(d-2)} \frac{R}{\lambda D} k^{d-2},
$$

where $R$ is a positive Ricci scalar curvature of the target manifold with dimension $[\text { mass }]^{2}$. By using $d=4+\epsilon$ expansion and the minimal subtraction scheme, we obtain a regularized function

$$
\delta_{Z}(k)=C_{d} \frac{R}{\lambda D} k^{d-2}+C,
$$


where $C_{d}^{-1}=(4 \pi)^{d / 2} \Gamma(d / 2+1)$ and $C$ represents an integral constant taking the value $\delta_{Z}(0)$ to be determined by the initial renormalization condition Eq.(20). Thus for $d=4$ the renormalization function in the IR region behaves like

$$
\delta_{Z}(k)=\frac{1}{128 \pi^{2}} \frac{R}{\lambda} k^{2}+C, \quad(\text { for small } k) .
$$

To determine the integral constant $C$ we need to apply the initial renormalization condition defined at UV. However, the function Eq.(23) is only valid at IR, obvious it diverges in the limit $k \rightarrow \infty$. To a large extent, the difficulty can be attributed to the fact that such behavior of $\delta_{Z}$ will completely change in the UV region as a consequence of the running of the effective dimension of the system. In fact, the scale dependent anomalous dimension $\eta(k)$ always go with dimension $d$ and produces a scale dependent effective $\epsilon$-parameter (expansion) of the dimensional continuation, in other words, everywhere that appears the dimension $d$ could be approximately replaced by $d_{e f f}=d-\eta(k)$. Since the anomalous dimension equals to $\eta(k \rightarrow \infty)=d-2$ at the non-trivial UV fixed point [19], the effective dimension near the UV fixed point tends to $d_{\text {eff }}=d-\eta(k \rightarrow \infty)=2$, but rather 4 , and hence in the UV region, the power of $k$ goes to zero in Eq.(21), and $\delta_{Z}$ is expected to behave as $\log k$ instead. One could see that the dimension $d$ and the behavior of $\delta_{Z}(k)$ are very different for small $k$ and large $k$ region,

$$
d_{\text {eff }}=\left\{\begin{array}{ll}
4 & (k \rightarrow 0) \\
2 & (k \rightarrow \infty)
\end{array}, \quad \delta_{Z} \sim\left\{\begin{array}{ll}
k^{2} & (\text { for small } k) \\
\log k & (\text { for large } k)
\end{array} .\right.\right.
$$

We can see that the growth rate $\partial \delta_{Z} / \partial k$ is always positive, which means $\delta_{Z}$ grows and the coupling decreases with the increasing of $k$. However, it does not mean the theory is asymptotically free, it is in fact "asymptotically safe". The reason is transparent, although at IR $\delta_{Z}$ grows as $\sim k^{2}$, the rate slows down to $\sim(d-2) k^{d-2}$ in the UV region, and finally the growth rate vanishes as $d \rightarrow 2$ and the coupling approaches to a finite value at the UV fixed point.

The dimension reduction mechanism from $4 \mathrm{~d}$ to $2 \mathrm{~d}$ in gravitational system is critical for the non-perturbative renormalizability and the existence of a non-Gaussian fixed point which is suggested by literature, e.g. asymptotically safe gravity. The difference between those and ours is that it is the dimension reduction of $d$ of the base space of the NLSM but the physical dimension $D$ of the target space, although they are related in the semi-classical approximation. The dimension reduction of the base space of NLSM is purely a renormalization effect which has a simple fractal geometry interpretation [21]. Because of such mechanism, the proposed theory is very different from conventional 2d NLSM which is effectively the UV limit of our theory, while in the IR region the theory behaves like a 4d NSLM. For this reason, the asymptotic UV critical behavior of the theory does not explicitly depend on the dimension $d$ of the base space. The qualitative behavior of the theory is determined not by the explicit fundamental Lagrangian, but rather by the nature of the basic symmetry $O(D)$ or $O(1, D-1)$, where $D$ is the only parameter, that are imposed on the family of Lagrangian with different $d$ that flow into one another, and by the universal nature of the UV fixed point.

In the UV region or small scale, the classical notion of metric and curvature may become improper, a viable approach to probe the region may be to regard the NLSM as an equivalent constrained linear theory Eq.(9) without these geometric notion, from the action at that scale where the dimension reduced to $d=2$, the renormalization function can be given by

$$
\delta_{Z}(k)=D \int_{\Lambda \leq|p|<k} \frac{d^{2} p}{(2 \pi)^{2}} \frac{i}{p^{2}},
$$

in which $\Lambda$ is certain renormalization scale where the dimension is effectively reduced. We obtain the integral for the UV cutoff $k$,

$$
\delta_{Z}(k)=\frac{D}{2 \pi} \log \frac{k}{\Lambda}+C, \quad(\text { for large } k) .
$$

which is a counterpart of Eq.(23) for large $k$ when the dimension is sufficiently reduced to $d_{\text {eff }}=2$.

In order to determine the k-independent constant $C$, we make use of the continuity and universality of the scaling dimension $\gamma$ function in renormalization. For small $k$ where $d_{e f f}=4$, by using Eq.(23) the $\gamma$ function is given by

$$
\gamma(k)=\frac{1}{2} k \frac{\partial \delta_{Z}}{\partial k}=\frac{1}{128 \pi^{2}} \frac{R}{\lambda} k^{2}, \quad(\text { for small } k),
$$

while for large $k$ where $d_{\text {eff }} \rightarrow 2$, by using Eq.(26) it changes from the quadratic behavior to a universal critical scaling dimension

$$
\gamma_{c}=\gamma(k \rightarrow \infty)=\frac{1}{2} k \frac{\partial \delta_{Z}}{\partial k}=\frac{D}{4 \pi}, \quad(\text { for large } k)
$$


Since $\gamma$ is a smooth function of $k$ and, in the asymptotic UV region, it continuously approaches to the universal value independent to the cutoff $k$, they must be identical at certain large $k_{i d}$,

$$
\gamma\left(k_{i d}\right)=\frac{1}{128 \pi^{2}} \frac{R}{\lambda} k_{i d}^{2}=\frac{D}{4 \pi}
$$

At such large scale $k_{i d}$ where the $\gamma$ function becomes universal and hence the scaling of the system starts becoming critical, it is safe to consider the initial renormalization condition Eq.(20) starts applying. Substituting the Eq.(29) into Eq.(23) we have

$$
\delta_{Z}\left(k_{i d}\right)=\frac{D}{4 \pi}+C=0
$$

which immediately demands a universal value of $C$ relating to the universal critical scaling $\gamma_{c}$ at $\mathrm{UV}$, by using $D \equiv 4$,

$$
C=-\gamma_{c}=-\frac{1}{\pi}
$$

Despite the above result is evaluated under the one-loop approximation, to some extent the general result that the constant $C$ is exactly the minus critical scaling $-\gamma_{c}$ could be seen reliable independent to any approximation method. Because in the vicinity of the UV fixed point, the system becomes nearly critical, the renormalization function $Z(k)$ is nothing but a power behavior

$$
Z(k \rightarrow \infty) \sim\left(\frac{k^{2}}{M^{2}}\right)^{\gamma(k)+C} \quad, \quad(\text { for large } k) .
$$

Therefore, by applying the initial renormalization condition, $Z(k \rightarrow \infty)=1$, the exponent $\gamma(k)+C$ has to be exactly vanish at $\mathrm{UV}$, so it must have $C=-\gamma_{c}$.

At this moment, we arrive at an asymptotic function $\delta_{Z}$ in the IR region, which is able to extrapolatively satisfy the initial renormalization condition at UV,

$$
\delta_{Z}(k)=-\frac{1}{\pi}+\frac{1}{128 \pi^{2}} \frac{R}{\lambda} k^{2}, \quad(\text { for small } k) .
$$

For this formula, we should not incorrectly consider that $k$ must stop at a particular point $k_{c}$ to impose the renormalization condition $\left(\delta_{Z}\left(k_{c}\right)=0\right)$. We must emphasis that $k$ will not stop at $k_{c}$, it could continuously go to infinity, since at large $k$ the behavior of $\delta_{Z}$ changes as the effective dimension approaches 2 but rather 4 , and $\delta_{Z}$ increases as log $k$ but rather $k^{2}$. We know that the NLSM near $d=2$ becomes perturbative renormalizable [22-24] which is a positive feature for a good behavior of our model at UV. And finally the increasing rate slows down and stop at the UV fixed point, where the $\delta_{Z}$ vanishes as the renormalization condition imposes, leaving the finite bare $\lambda$. In this sense, the theory truly has a non-trivial UV fixed point, where the theory is well-defined at quantum level with finite number of relevant bare inputs.

On the other hand, note that the critical scaling dimension at UV affects the IR behavior non-trivially. As $k \rightarrow 0$, $Z(k)$ changes from unity at UV limit to $1-\gamma_{c}$ at IR limit. As a consequence, the theory also has a non-trivial IR fixed point, the $\lambda$ is renormalized to be a finite value at IR,

$$
\lambda_{I R}=\left(1-\frac{1}{\pi}\right) \lambda \approx 0.68 \lambda .
$$

Note that the $\lambda_{I R}$ is of the same order of the UV bare value $\lambda$, so the perturbation technique is not only valid for UV but also IR. In this sense, we consider the above results done for small $k$ are also reliable. As we will discuss later, the non-trivial IR fixed point is very crucial for understanding the cosmic observations, such as accelerating expansion and cosmological constant.

\section{B. Effective Action: Emergent Cosmological Constant}

In this subsection, we interpret the effective theory and discuss its relation to the cosmic observations. Substituting the Eq.(32) into Eq.(19), taking $d=4$, one get the effective action in the IR region,

$$
S_{k}=\frac{1}{2} \int d^{4} x\left(\lambda-\frac{1}{\pi} \lambda+\frac{1}{128 \pi^{2}} R k^{2}\right) g_{\mu \nu} \partial_{a} X^{\mu} \partial_{a} X^{\nu},
$$


in which the parameter $\lambda$ is a $x$-independent constant and hence can be taken into the integral. By definition $\lambda$ is a $k$-independent UV bare value, and relates to a UV scale $k_{U V}$ and a fixed point value of Ricci scalar curvature [19]. Perturbative calculation shows that it is of order $\lambda \sim \mathcal{O}\left(R_{*} k_{U V}^{2}\right)$, where $R_{*}$ is a Ricci scalar curvature at a fixed point that can be evaluated by a Ricci-type flow equation. To find the precise value of $\lambda$, without loss of generality, we can re-parameterize it as $\lambda=\frac{\zeta R_{*}}{32 \pi G}$ where the numerical factor $\zeta$ is to-be-determined, and $G$ is the Newton's constant playing the role of the $k_{U V}^{-2}$. The action is rewritten as

$$
S_{k}=\frac{1}{4} \zeta \int d^{4} x\left(\frac{R_{*}}{16 \pi G}-\frac{2}{\pi \zeta} \lambda+\frac{1}{64 \pi^{2} \zeta} R k^{2}\right) g_{\mu \nu} \partial_{a} X^{\mu} \partial_{a} X^{\nu}
$$

Remind the renormalization condition that near the UV fixed point the second term in the parenthesis is nearly canceled by the asymptotic UV form of the third term, leaving the first term near the UV fixed point an EinsteinHilbert-like term, in which only the Ricci curvature being a fixed point Ricci curvature $R_{*}$. By comparison of the pre-factor with that in the semi-classical action Eq.(15), one has $\zeta=1$. In this sense, the first term $\lambda$ should be interpreted as Einstein-Hilbert term at a fixed point, and the second term $(1 / \pi) \lambda$ should be interpreted as a cosmological constant term.

Under a renormalization flow equation, a Ricci curvature $R$ flows to a homogeneous constant curvature $R_{*}$ at the fixed point. Thus $R_{*}$ or $\lambda$ is a k-independent constant describing a homogeneous curvature background of the target manifold, the third k-dependent renormalization correction term describes a curvature fluctuation upon the background. At certain renormalization scale, the first and third terms in the parenthesis of Eq.(35) together reproduce an effective k-dependent Einstein-Hilbert term in terms of Ricci curvature $R$. Consequently, the homogeneous $R_{*}$ is given by a renormalization flow from a locally inhomogeneous Ricci curvature,

$$
R=R_{*}\left(1-\frac{1}{4 \pi} G k^{2}\right)^{-1}, \quad(\text { for small } k),
$$

in which we find that $R_{*}$ is in fact an IR fixed point $(k=0)$ value of $R$. From this flow equation of Ricci curvature, we can see that a locally inhomogeneous $R$ in the standard Einstein-Hilbert term is reproduced from the renormalization effect. At larger and larger spacetime scale, an inhomogeneous Ricci curvature $R$ is smoothed out and converges to a constant background curvature $R_{*}$, or equivalently speaking, a locally inhomogeneous Einstein-Hilbert term is reproduced from a smooth background $R_{*}$ plus quantum fluctuations.

At this moment, we could substitute the effective action of reference frame Eqs. $(35,36)$ into the Eq.(12) and using the semi-classical approximation to treat $X_{\mu}$, then obtain a total effective action formulated by using the internal and physical coordinates $X_{\mu}$,

$$
S_{e f f}\left[\varphi, g_{\mu \nu}\right]=\int d^{4} X \sqrt{\operatorname{det} g}\left[\frac{1}{2} g^{\mu \nu} \frac{\delta \varphi}{\delta X^{\mu}} \frac{\delta \varphi}{\delta X^{\nu}}-V(\varphi)+\frac{R}{16 \pi G}-\frac{2}{\pi} \lambda\right] .
$$

The first two terms in the bracket are the ordinary matter term, the third term is interpreted as the Einstein-Hilbert term at certain scale. The fourth term $(2 / \pi) \lambda$ is a positive constant related to the IR fixed point Ricci curvature being of order $R_{*} k_{U V}^{2}$, which here is interpreted as the effective cosmological constant corresponding to an effective energy density

$$
\rho_{\Lambda}=\frac{2}{\pi} \lambda
$$

Note that the critical density is defined by $\rho_{c}=\frac{3 H^{2}}{8 \pi G}$, where $H$ is the Hubble parameter. The value of near current epoch $H_{0}=\left.H\right|_{z \sim 0}$ then gives $\left.\rho_{c}\right|_{z \sim 0}=\frac{3 H_{0}^{2}}{8 \pi G}$, which is a density averaged by a volume with respect to rulers and clocks at near current epoch. By using the relation between the homogeneous Ricci curvature background and curvature radius $r, R_{*} \equiv D(D-1) r^{-2}=12 r^{-2}$, where the curvature radius $r$ is given by the current Hubble parameter $r=H_{0}^{-1}$, so one find that the critical density is just the UV fixed point value of $\lambda$,

$$
\left.\rho_{c}\right|_{z \sim 0}=\lambda=\frac{R_{*}}{32 \pi G} .
$$

Then we have $\left.\rho_{\Lambda} \doteq(2 / \pi) \rho_{c}\right|_{z \sim 0}$, where " $\doteq$ " stands for the neglecting of the renormalization correction. It is an interesting result that the dark energy is equal to $2 / \pi$ times the "current" critical density,

$$
\left.\Omega_{\Lambda}\right|_{z \sim 0}=\frac{2}{\pi} \approx 0.64
$$


which agrees with the observations well. The word "current" means that the density is averaged by the volume relative to the scales of rulers and clocks near $z \sim 0$. Certainly, $\rho_{\Lambda} \doteq(2 / \pi) \lambda$ and $\left.(2 / \pi) \rho_{c}\right|_{z \sim 0}$ are just equal in values, they are not really identical. $\rho_{\Lambda} \doteq(2 / \pi) \lambda \propto R_{*}$ is a scalar, it gives rise to a full stress tensor $T_{\mu \nu}=\rho_{\Lambda} g_{\mu \nu}$, for this reason, its equation of state is exactly $w=-1$, in contrast, $\rho_{c}$ is just the $T_{00}$ component of a stress tensor, so they behave differently under spacetime coordinates transformation. It is easy to see that $\rho_{\Lambda} \doteq(2 / \pi) \lambda \propto R_{*}$ is invariant with respect to the physical clock time, in this sense it is a constant, but $(2 / \pi) \rho_{c}$ varies with the redshift, they are just equal in values near $z \sim 0$.

In the framework only the concepts defined in a relative way, but rather absolute, are observable. A physical observable is redshift $z$, while the absolute global age of the universe is essentially unobservable in our theory. In this sense, discussions about the universe evolution should not base on the global age but the redshift. In other words, the quantities being functions of the global age, e.g. $H(t), \rho_{i}(t), \Omega_{i}(t)$ should be replaced by some more physical ones being functions of the redshift: $H(z), \rho_{i}(z), \Omega_{i}(z)$. It is no problem that the near current epoch $z \sim 0$ always exist in every epoch of the expansion history of the universe, leading to the consequence that an observer at his/her near "current" epoch "always" find $\left.\Omega_{\Lambda}\right|_{z \sim 0} \approx 0.64$ no matter what is the absolute epoch he/she lives. In this sense, $\rho_{\Lambda}$ is "always" comparable with $\left.\rho_{c}\right|_{z \sim 0}$ seeing by observers at his/her epoch. The word "always" is about the relational redshift but rather the absolute history or age, meaning that the value of $\Omega_{\Lambda}$ is fixed with respect to the observers at $z \sim 0$, it does not mean $\rho_{\Lambda}$ and $\rho_{c}$ behave in the same way in the expansion history of the universe. As we know, they behave differently about the redshift, $\rho_{c}(z)$ increases with $z$, while $\rho_{\Lambda}$ does not change, resulting to that $\Omega_{\Lambda}(z)=\rho_{\Lambda} / \rho_{c}(z)$ decreases as redshift increases. The framework gives a non-dynamical explanation to the coincidence problem, namely why the dark energy is comparable with the critical density now? The key to understand the coincidence is to use the relational observable (such as redshift) independent to any absolute scale of the universe, avoiding using the quantity such as the global age of the universe defined by an absolute observer looking from its outside. We will also come back to the coincidence problem in discussing the distance-redshift relation in the next subsection.

\section{Physical Interpretation: Spacetime Uncertainties at Cosmic Distance}

We have seen that the renormalization function $\delta_{Z}$ or $Z$ is crucial in understanding the cosmological constant. In this subsection, we will discuss its physical interpretation.

The previous renormalization results can also be understood by the mean field method. In such language, providing that $\Delta X_{\mu}(x)$ is a displacement between $X_{\mu}(x)$ and a given point $X_{\mu}(0)$, i.e. $\Delta X_{\mu}(x)=X_{\mu}(x)-X_{\mu}(0)$. The renormalized quantity $\Delta X_{\mu}^{r e}$ can be expanded around the mean field value $\left\langle\Delta X_{\mu}\right\rangle$ by a quantum fluctuation $\delta X_{\mu}$,

$$
\Delta X_{\mu}^{r e}=\left\langle\Delta X_{\mu}\right\rangle+\delta X_{\mu}
$$

By using this relation and Eqs. $(18,19)$, considering that the vacuum expectation value of the fluctuation vanishes $\left\langle\delta X_{\mu}\right\rangle=0$, we find that $\delta_{Z}(k)$ is nothing but a measure of a ratio of each variance to the mean-squared of its spacetime coordinate difference,

$$
\delta_{Z}(k)=Z(k)-1=-\frac{1}{2} \frac{\left\langle\delta X_{\mu}^{2}\right\rangle}{\left\langle\Delta X_{\mu}\right\rangle^{2}}
$$

Recall the result Eq.(32), we have

$$
\frac{\left\langle\delta X_{\mu}^{2}\right\rangle}{\left\langle\Delta X_{\mu}\right\rangle^{2}}=-2 \delta_{Z}=\frac{2}{\pi}\left(1-\frac{1}{4} G k^{2}\right), \quad(\text { for small } k) .
$$

This formula indicates an inescapable and universal quantum limitation to the spacetime accuracy at IR. One can not have rulers and clocks precisely measured and synchronized across spacetime. In the IR limit, or equivalently, at cosmic scale, the ratio of the variance $\left\langle\delta X_{\mu}^{2}\right\rangle$ to the mean-squared of spacetime distance $\left\langle\Delta X_{\mu}\right\rangle^{2}$ is universal,

$$
\lim _{k \rightarrow 0} \frac{\left\langle\delta X_{\mu}^{2}\right\rangle}{\left\langle\Delta X_{\mu}\right\rangle^{2}}=\frac{2}{\pi} .
$$

The universality of the ratio is closely related to the universality of the critical dimension $\gamma_{c}$ in Eq.(31). In the next subsection, we will see that the universality of the ratio is also a natural consequence of the generalized equivalence principle.

The relation Eq.(44) for the variance $\left\langle\delta X_{\mu}^{2}\right\rangle$ is valid at extreme IR or cosmic distance $\left\langle\Delta X_{\mu}\right\rangle^{2}$, which can be seen as an inherent cosmic variance of a quantum measurement at cosmic distance. It generalizes the result of refs. [4, 5] 
by putting the space and time on an equal footing. In those papers, only the uncertainty of a physical clock time field $X_{0}$ is considered, nevertheless the spatial coordinates are just treated as parameter background for the sake of simplicity. The difference between them is as follow. For the result in refs. $[4,5]$, the clock time variance grows linearly with the spatial distance and the proportional coefficient is the inverse of the spatial volume cutoff. However, in this paper the clock variance instead grows quadratically but linearly. Since the space and time is considered symmetric and isotropic, the clock variance can be interpreted as being proportional to the squared spatial distance. The reason for the different power dependence is due to the fact that, in the standard quantum mechanics, it is of first order in the derivative with respect to an evolution parameter, for instance the derivative with respect to the Schrodinger's time or the renormalization scale $\log k$, but in a theory that space and time are put on an equal footing, for instance a relativistic theory, the orders in the derivatives with respect to spatial and temporal distance are the same, for bosonic degrees of freedom, they are both of second order.

For the reason that time or frequency can be conveniently measured by redshifts of distant spectral lines in cosmic observations, they could be used as idea clocks distant from us to test the effect at cosmic distance. The clock time uncertainty can also be interpreted in terms of a redshift uncertainty or broadening. Although many physics affect the redshift broadening such as thermal fluctuation, a prediction may be testable: the ratio of the inherent quantum variance of redshift to its mean-squared equals

$$
\lim _{k \rightarrow 0} \frac{\left\langle\delta z^{2}\right\rangle}{\langle z\rangle^{2}}=\frac{2}{\pi}
$$

which is derived from Eq.(44) by using $\left\langle\delta z^{2}\right\rangle=\left\langle\delta X_{\mu}^{2}\right\rangle /\left\langle X_{\mu}(0)\right\rangle^{2}$ and $\langle z\rangle=\left\langle X_{\mu}(x)-X_{\mu}(0)\right\rangle /\left\langle X_{\mu}(0)\right\rangle$ if we consider $\left\langle X_{\mu}(x)\right\rangle$ measures certain characteristic wavelength or period at the given point $x$. The result is also deduced in ref.[5]. The $\left\langle\delta z^{2}\right\rangle$ does not change the mean value $\langle z\rangle$, but shifts the mean-squared

$$
\left\langle z^{2}\right\rangle=\langle z\rangle^{2}+\left\langle\delta z^{2}\right\rangle \stackrel{k \rightarrow 0}{=}\left(1+\frac{2}{\pi}\right)\langle z\rangle^{2} .
$$

Therefore, the distance-redshift relation $D(z)$ is modified at order $\mathcal{O}\left(z^{2}\right)$ by this effect [5]. The first order $\mathcal{O}(z)$ term of $D(z)$ relates to the expansion rate of the universe, and the second order $\mathcal{O}\left(z^{2}\right)$ term relates to an accelerating or decelerating of the expansion. More precisely, the universal ratio Eq.(45) contributes an additional deceleration parameter $q_{0}=-2 / \pi$, which is redshift independent and uniform, to the distance-redshift relation besides other components of the universe. It makes a departure to the Hubble's law which is more significant at high redshift. Expanding the expectation value of the luminosity distance $\langle D(z)\rangle$ in powers of redshift to the second order [25], we have

$$
\langle D(z)\rangle=\frac{1}{H_{0}}\left[\langle z\rangle+\frac{1}{2}\left(1+\frac{2}{\pi}+\ldots\right)\langle z\rangle^{2}+\mathcal{O}\left(\langle z\rangle^{3}\right)\right],
$$

where the additional positive constant $2 / \pi$ plays the role of the percentage of dark energy $\Omega_{\Lambda}$, the $\ldots$ in the parenthesis represents the deceleration parameter coming from other components of the universe such as ordinary matter given by $-\Omega_{m}(1+z)^{3}$. $H_{0}$ is the Hubble constant measured at $z \sim 0$. The formula does not involve any absolute age of the universe, so for any observer, it is always valid no matter when the observer lives in the expansion history of the universe, the universe is always seen to become accelerating at near current epoch $z \approx 0.3$ at which the dark energy and the matter become comparable, i.e. $q_{0}=\Omega_{m}(1+z)^{3}-\Omega_{\Lambda}=0$. This fact also demonstrates the coincidence. The $\left\langle\delta z^{2}\right\rangle$ correction to the distance-redshift relation makes it become anomalous at high redshift, which is observed from high redshift supernovas being the first indication of the accelerating expansion of the universe [26, 27].

\section{Generalization of the Equivalence Principle}

In previous sections, we have shown that a correct value of cosmological constant and an effective Einstein-Hilbert term emerge in the IR region of the quantum dynamics of spacetime reference frame. From the dynamics of quantum spacetime reference frame to the concept of gravity, a further assumption is required: the equivalence principle. The principle gives a spacetime interpretation of gravity in classical general relativity, which is well established and tested in classical physics. However, the principle puzzles physicist when the quantum effects are seriously taken into account. Since the zero-point quantum fluctuation seems real (e.g. contributing to the Lamb shift), but why these large amount of energies of vacuum do not gravitate as the equivalence principle asserts, which is the main puzzle of the cosmological constant problem.

In fact the Lamb shift gravitates normally [28, 29], and there is no hint to assume that the energies coming from classical and quantum contributions produce different gravitational effects. In fact, only when the equivalence 
principle is valid, the accelerating expansion of the universe revealed by Eq.(47) is equivalent to the existence of a "dark energy" or a positive non-vanishing cosmological constant revealed by Eq.(37), so it seems like an indication that in our framework the equivalence principle could exactly hold. Furthermore, an elegant and economic assumption is also to maintain the spirit of relativity and hence the equivalence principle, so that gravity is just simply a relative phenomena, and there is nothing else more than that even at the quantum level. In this sense, the equivalence principle must be generalized to the quantum level to resolve the cosmological constant problem.

In this paper, we argue that the equivalence principle is also valid for all quantum phenomenon as it applies to the classical phenomenon. As the generalized equivalence principle claims, all kinds of energies including the quantum fluctuations gravitate. The generalized equivalence principle implies that all kinds of apparent curving of spacetime including those coming from quantum fluctuations or quantum uncertainties of spacetime are equivalent to gravitation, more precisely, the quantum uncertainties of spacetime deduced in this paper are equivalent to the accelerating expansion of the universe. Not only one can not distinguish gravitation from acceleration according to the classical equivalence principle, but also unable to distinguish gravitation from quantum spacetime intrinsic uncertainty/fluctuation. Therefore, the Ricci curvature in the effective action Eq.(37) can precisely be interpreted as gravity. The accuracy of the generalized equivalence principle can be demonstrated by the universality of the ratio Eq.(45) which is independent to the energies of the spectral lines. In other words, all spectral lines taking different energies uniformly "free-fall", it is not merely a particular property of the spectral lines, it is a universal property of the spacetime itself, so a universal accelerating expansion inevitably appears. The uncertainty/fluctuation of spacetime Eq.(44) or redshift Eq.(45) on the one hand can be interpreted as that the objects (spectral lines) are uniformly accelerating, or equivalently on the other hand, the spacetime is curved by the quantum fluctuation energy density $(2 / \pi) \lambda$ in Eq.(37) which is seen as a repulsive gravitational force.

To understand further how the generalized equivalence principle resolves the notorious problem of zero-point vacuum energies $\sum_{k} \frac{1}{2} \omega_{k}$ predicted in textbook quantum fields theory, we note that such zero-point vacuum energies are not involved in our effective theory Eq.(37). The effective vacuum energies density or the cosmological constant as a source of gravity in Eq.(37) comes from the two-point function $\left\langle\delta X_{\mu} \delta X_{\nu}\right\rangle \neq 0$, while the vacuum expectation value vanishes, $\left\langle\delta X_{\mu}\right\rangle=0$. In other words, the energies of quantum fluctuations of spacetime relating to two states are the leading contribution to the vacuum energies and gravitational effects [30, 31], which do obey the generalized equivalence principle. However, the notorious zero-point vacuum energies relating to one state have no gravitational effects. Since quantum fluctuation of a physical reference frame is inevitable, fundamentally speaking, the absolute rest frame can not be precisely realized at the quantum level. For this reason, the zero-point vacuum energies which make sense only with respect to a perfect classical absolute rest frame in textbook quantum mechanics are completely unphysical and unobservable in any laboratory experiment, including the Casimir effect [32]. This property resolves the first part of the cosmological constant problem, namely, why the zero-point vacuum energies do not gravitate. The physical energies which gravitate normally are those make sense with respect to the quantum spacetime reference frame that is also subject to quantum fluctuation. As an application, the dark energy from the vacuum fluctuation $\left\langle\delta X_{\mu} \delta X_{\nu}\right\rangle$, which relative to each other, gravitate normally as the generalized equivalence principle asserts. This property solves the second part of the cosmological constant problem, namely, why the cosmological constant is so small.

\section{SUMMARY AND CONCLUSIONS}

To solve the cosmological constant problem, the quantum dynamics and the effects of a quantum clock can not be neglected. In this paper, acting on the spirit of treating space and time on an equal footing, we generalize the quantum clock to the quantum spacetime reference frame, via a physical realization of a reference system by quantum rulers and clocks. It is in this sense we have a "quantum spacetime" obeying both quantum mechanics and general relativity.

In order to accommodate quantum mechanics to general relativity, the textbook quantum mechanics must be generalized. General relativity is general covariant or observer independent, a physical quantum state satisfying this property is in general an entangled state entangling a to-be-studied quantum physical system with a quantum measuring device. It is necessary to have a relational interpretation to the entangled state, since the to-be-studied system makes sense only relative to the quantum measuring device. Entangled state solves the diffeomorphism constraints and the Wheeler-DeWitt equation, which plays a more fundamental role than the textbook Schrodinger equation. Since clock time is inescapably subject to quantum fluctuation, the Schrodinger equation using the parameter time is just an approximation. The cosmological constant problem is an indication of going beyond the Schrodinger equation where the quantum fluctuation of time is inescapable and must not be ignored.

Omitting the internal degrees of freedom of the physical rulers and clocks, such as their spins, considering only their metric properties, the spacetime reference frame is described by the bosonic non-linear sigma model. In a semiclassical treatment of the spacetime reference frame, we recover the existing theories: quantum fields theories in fixed 
curved spacetime. In a complete quantum treatment, we studied its normalization behavior under approximations. The theory has a non-trivial UV fixed point, namely it is asymptotically safe, and hence the notion of spacetime still makes sense even at UV. We get three surprising results from the theory. The first, and most remarkable, result is that a cosmological constant appears which naturally gives not only a correct order but also a percentage $\Omega_{\Lambda}=2 / \pi \approx 0.64$ very close to current observations. The second result is that the quantum dynamics of the quantum spacetime automatically contains an effective Einstein-Hilbert action, and hence automatically incorporates a theory of gravity under the assumption of the validity of equivalence principle at the quantum level. The third result says that the spacetime are inescapably subject to quantum uncertainties, one can not have rulers and clocks perfectly measured and synchronized across spacetime. The ratio of the variance to the mean-squared of the spacetime distance tends to a universal constant $\left\langle\delta X_{\mu}^{2}\right\rangle /\left\langle\Delta X_{\mu}\right\rangle^{2}=2 / \pi$ in the extreme IR region of theory. We also argue that this effect is testable by observing a linear dependence between the inherent quantum variance and mean-squared of redshifts from distant spectral lines. The proportionality is $\mathcal{O}(1)$ and expected to be identical to the percentage of the dark energy $\Omega_{\Lambda}$. These results strongly support the argument that the equivalence principle still holds at the quantum level. It is in this sense we propose a possible "quantum theory of gravity".

\section{Acknowledgments}

This work was supported in part by the National Science Foundation of China (NSFC) under Grant No.11205149, and Science Research Foundation of Jiangsu University under Grant No.15JDG153.

[1] S. Weinberg, Rev. Mod. Phys. 61, 1 (1989).

[2] R. Bousso, General Relativity and Gravitation 40, 607 (2008), 0708.4231.

[3] J. Martin, Comptes Rendus Physique 13, 566 (2012), 1205.3365.

[4] M. J. Luo, Nuclear Physics B 884, 344 (2014), 1312.2759.

[5] M. J. Luo, Journal of High Energy Physics 6, 63 (2015), 1401.2488.

[6] Planck Collaboration, P. A. R. Ade, N. Aghanim, C. Armitage-Caplan, M. Arnaud, M. Ashdown, F. Atrio-Barandela, J. Aumont, C. Baccigalupi, A. J. Banday, et al., ArXiv e-prints (2013), 1303.5062.

[7] Y. Aharonov and T. Kaufherr, Phys. Rev. D 30, 368 (1984).

[8] C. Rovelli, Classical and Quantum Gravity 8, 317 (1991).

[9] M. Dickson, Studies in History and Philosophy of Science Part B: Studies in History and Philosophy of Modern Physics 35, 195 (2004)

[10] R. M. Angelo, N. Brunner, S. Popescu, A. J. Short, and P. Skrzypczyk, Journal of Physics A: Mathematical and Theoretical 44, 145304 (2011).

[11] D. N. Page and W. K. Wootters, Phys. Rev. D 27, 2885 (1983).

[12] E. Moreva, G. Brida, M. Gramegna, V. Giovannetti, L. Maccone, and M. Genovese, Phys. Rev. A 89, 052122 (2014), 1310.4691

[13] C. Rovelli, International Journal of Theoretical Physics 35, 1637 (1996), quant-ph/9609002.

[14] C. Rovelli, Quantum gravity (Cambridge University Press, 2004).

[15] N. G. Phillips and B. Hu, Physical Review D 62, 084017 (2000).

[16] M. Gell-Mann and M. Lévy, Il Nuovo Cimento 16, 705 (1960).

[17] S. V. Ketov, Quantum non-linear sigma-models: from quantum field theory to supersymmetry, conformal field theory, black holes and strings (Springer Science \& Business Media, 2000).

[18] J. Zinn-Justin, Quantum field theory and critical phenomena (Oxford University Press, Oxford, 2002).

[19] A. Codello and R. Percacci, Physics Letters B 672, 280 (2009).

[20] S. Weinberg, in S. W. Hawking and W. Israel (Eds), General relativity: An Einstein centenary survey (Cambridge University Press, 1979).

[21] H. Kroger, Elsevier Science Publishers B.v.amsterdam pp. i-vi,799-928 (2002).

[22] E. Brezin, J. Zinn-Justin, and J. Le Guillou, Physical Review D 14, 2615 (1976).

[23] D. Friedan, Physical Review Letters 45, 1057 (1980).

[24] D. Friedan, Annals of Physics 163, 318 (1980).

[25] S. Weinberg, Cosmology (Oxford University Press, Oxford, 2008).

[26] A. G. Riess et al. (Supernova Search Team), Astron.J. 116, 1009 (1998), astro-ph/9805201.

[27] S. Perlmutter et al. (Supernova Cosmology Project), Astrophys.J. 517, 565 (1999), astro-ph/9812133.

[28] J. Polchinski, ArXiv High Energy Physics - Theory e-prints (2006), hep-th/0603249.

[29] E. Massó, Physics Letters B 679, 433 (2009), 0902.4318.

[30] T. Padmanabhan and T. Singh, Classical and Quantum Gravity 4, 1397 (1987).

[31] T. Padmanabhan, Classical and Quantum Gravity 22, L107 (2005), hep-th/0406060. 
[32] R. L. Jaffe, Phys. Rev. D 72, 021301 (2005). 